\title{
Fracture Resistance of Endodontically Treated Teeth Restored Using Three Different Esthetic Post Systems
}

Vibha Hegde ${ }^{1}$, Nikita Arora ${ }^{2}$

\begin{abstract}
Aim: To compare and evaluate the fracture resistance of endodontically treated teeth restored with Rebilda post GT, EverStick post and prefabricated glass fiber post at 90-degree vertical load and 45-degree oblique load.

Materials and methods: Eighty freshly extracted single-rooted mandibular premolars were selected, and after root canal preparation and obturation, standardized post spaces were prepared. Samples were randomly divided into four groups $(n=20)$ depending on the type of restorative technique used: group I restored with Rebilda post GT system (bundled glass fiber reinforced composite post), group II restored with EverStick post (Individually formable glass fiber root canal post), group III restored with prefabricated glass fiber post, group IV restored with direct composite resin restoration without a post (control). Using a universal testing machine, 90 -degree vertical and 45 -degree oblique load was applied to the restored teeth with a crosshead speed of $0.5 \mathrm{~mm} /$ minute. Fracture loads and mode of fracture was recorded.

Results: The results of the analysis of variance (ANOVA) and post hoc Tukey HSD test revealed that the fracture resistance was significantly affected by different post systems $(p<0.001)$.
\end{abstract}

Conclusion: Rebilda post GT samples showed maximum fracture resistance followed by the EverStick fiber post group, prefabricated post, and least in the control group.

Keywords: Endodontically treated tooth, Fiber post, Fracture resistance, Rebilda post GT.

Journal of Operative Dentistry and Endodontics (2019): 10.5005/jp-journals-10047-0066

\section{INTRODUCTION}

Restoration of the mutilated endodontically treated tooth (ETT) is a subject that has been evaluated and discussed widely in dental literature. Esthetic, functional, and structural rehabilitation of a pulpless tooth is critically important to ensure a successful restorative outcome. Endodontically treated teeth present with dehydration, altered esthetic, and change in physical characteristics. ${ }^{1}$ These include decreased proprioception and moisture, leading to reduced dentin fracture resistance. ${ }^{2}$ According to Dietschi et al., ${ }^{3}$ the consequences of these changes are negligible. The major issues of ETT are related with the coronal destruction resulting from caries, dentin loss due to the removal of the roof of the pulp chamber, ${ }^{4}$ and the weakening of the pericervical dentin during access preparation. ${ }^{5}$ As a result of the compromised structural integrity, an increased fracture tendency during normal function is noted. ${ }^{6}$ Thus, in most ETT with inadequate coronal tooth structure, the use of intraradicular posts is recommended to encourage the retention of the final restoration and to biomechanically buttress the remaining tooth structure, thereby providing a coronoradicular stabilization. ${ }^{7,8}$ Development and use of fiber-reinforced composite (FRC) root canal posts have increased rapidly over the past few years. ${ }^{9}$

Glass fiber posts have an elastic modulus similar to that of dentin, ${ }^{10}$ consequently reinforcing the tooth structure. ${ }^{11}$ This property has been reported to reduce catastrophic root fracture and provide better stress distribution. ${ }^{12}$ Glass fiber post provides the most esthetic results and transmits light, ${ }^{13}$ require less dentin removal, and can be bonded to dentin. ${ }^{8}$

To overcome the difficulties that irregular root canal forms pose, an elastic FRC post (EverStick post, GC Europe, Leuven) was introduced to the market in 2011. This post is individually adaptable glass fibers, and its bonding and flexural properties (flexural strength: $1145 \mathrm{MPa}$, Young-modulus: $15 \mathrm{GPa})^{14}$ appear to be superior to commercially available, prefabricated FRC posts. ${ }^{9}$

\begin{abstract}
1,2 Department of Conservative Dentistry and Endodontics, YMT Dental College and Hospital, Navi Mumbai, Maharashtra, India

Corresponding Author: Nikita Arora, Department of Conservative Dentistry and Endodontics, YMT Dental College and Hospital, Navi Mumbai, Maharashtra, India, Phone: +91 9833699698, e-mail: na199320@gmail.com
\end{abstract}

How to cite this article: Hegde V, Arora N. Fracture Resistance of Endodontically Treated Teeth Restored Using Three Different Esthetic Post Systems. J Oper Dent Endod 2019;4(1):10-13.

Source of support: Nil

Conflict of interest: None

Rebilda post GT (VOCO GmbH, Germany) is a bundled glass FRC post. It consists of a bundle of fine individual posts $(0.3 \mathrm{~mm}$ in diameter) in varying numbers. Once the sleeve is removed, the bundle is spread, and the fine individual posts are distributed in the entire root canal. In contrast to conventional root posts, this provides homogeneous reinforcement of the entire core buildup.

This study hence attempts to evaluate the fracture resistance of endodontically treated teeth restored with Rebilda post, EverStick post, and prefabricated post at 90 -degree vertical and 45 -degree oblique load (Fig. 1).

\section{Materials and Methods}

Freshly extracted intact human mandibular premolars (for orthodontic purpose) without caries, with anatomically similar roots were selected. The teeth were examined with a stereomicroscope under 10x magnification to detect craze lines or cracks, which were excluded from the study, resulting in 80 specimens. Teeth were stored in $0.1 \%$ thymol solution and were decoronated at the cementoenamel junction (CEJ) with a diamond-coated saw (Isomet 2000; Buehler 


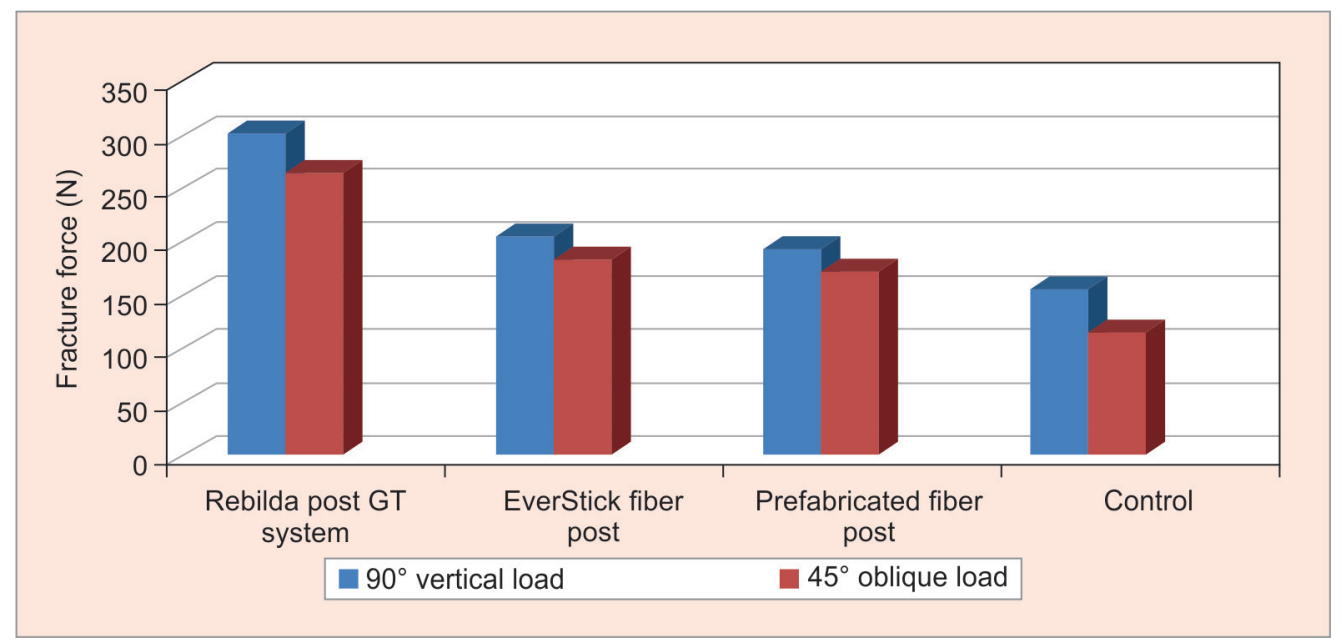

Fig. 1: Comparison of fracture resistance of different post systems at 90-degree vertical load and 45-degree oblique load

Ltd., Lake Bluff, Illinois, USA). The roots were adjusted to $16 \mathrm{~mm}$ in length, and the working length was established $1 \mathrm{~mm}$ short of the apex. All the root canals were instrumented using Protaper Universal rotary instruments till sizes F3 using crown-down technique under copious irrigation with $5 \mathrm{~mL}$ of $2.5 \%$ sodium hypochlorite $(\mathrm{NaOCl})$. Canals were rinsed with $1 \mathrm{~mL}$ of $17 \%$ aqueous ethylene diamine tetraacetic acid (EDTA) for 1 minute followed by distilled water as the final irrigant, to remove any traces of remnant sodium hypochlorite and dried using paper points (Protaper ${ }^{\circledR}$ Universal Paper Points) sizes F3 matching the final finishing file, and obturated with gutta-percha using cold lateral condensation technique and AH Plus sealer (DeTrey Dentsply, York, Pennsylvania, USA). The root access was temporarily sealed with Cavit (3M ESPE, Seefeld, Germany). Samples were later stored in an incubator at $37^{\circ} \mathrm{C}$ and $100 \%$ humidity for 1-week.

After 1-week of incubation, post space was prepared with size 2 Peeso Reamer burs and fiber post drill to a depth of $10 \mathrm{~mm}$. During preparation of the canal, $5 \mathrm{~mm}$ of endodontic filling was left at the apex of each canal. All samples received a final rinse with $5 \mathrm{~mL}$ of distilled water, and the excess was removed using paper points (Protaper ${ }^{\circledR}$ Universal Paper Points).

Samples were randomly divided into four groups $(n=20)$.

\section{Group I}

Restored with Rebilda post GT. Post space was prepared by Rebilda post drill $(1.0 \mathrm{~mm})$, and $1.0 \mathrm{~mm}$ apical diameter with 6 -single posts under the red sleeve was selected. The post was pretreated with silane for 60 seconds and then air-dried. After irrigation and drying of the root canals, they were treated by Futurabond U (VOCO America Inc.) dual-cure universal adhesive for 20 seconds using a intraoral tip. The adhesive bond layer was dried using paper points. Rebilda post GT was coated with Rebilda dual-cure luting composite cement (VOCO America Inc.) and then inserted into the root canal filled with the cement. Prior to polymerization of the luting composite, the sleeve was removed, so that the individual posts can be fanned out throughout the canal using a suitable instrument. After the post reached the regulated length, cement was light-cured from above the post for 20 seconds. Light curing was performed using a light-emitting diode unit (Bluephase polywave LED, Ivoclar Vivadent, Schaan, Liechtenstein).

\section{Group II}

Restored with EverStick post. Post space was prepared by size 2 Peeso Reamer burs. After irrigation and drying of the root canals, they were treated by Futurabond U (VOCO) for 30 seconds. The fibers of EverStick post were bundled according to the thickness of the root canal using the lateral condensation method. This post was gently removed from root canal, and then light-cured. The light-cured post was cemented using Rebilda dual-cure luting composite. After the post reached the regulated length, cement was light-cured from above the post, perpendicular to the fiber for 20 seconds.

\section{Group III}

Restored with prefabricated post. After the preparation of the root canal same as group I and II, single prefabricated glass fiber post was cemented according to the manufacture's instructions. Glass fiber post was slowly inserted into the cement-filled root canal and light-cured for 20 seconds.

\section{Group IV (Control)}

Restored with a composite core.

In order to ensure the uniformity of the specimens, the composite resin core build-ups were standardized using cellulite core-forming matrices of the same size. All the specimens were maintained in $100 \%$ humidity, for 24 hours, at $37^{\circ} \mathrm{C}$.

\section{Mechanical Testing}

Fracture Resistance test was carried out by applying a load using the Instron Universal Testing Machine (Zwick, Germany). A 90-degree vertical and 45-degree oblique load was applied with a cylindrical plunger of a crosshead speed of $0.5 \mathrm{~mm} /$ minute until fracture, and the fracture loads were recorded. The vertical load was applied to the center of the occlusal surface, while the oblique load was applied to the center of the cusp beneath which the post was located. Mode of fracture was observed by visual inspection with the aid of transillumination.

\section{Statistical Analysis}

Descriptive statistics were expressed as means and standard deviation (SD) for each group (Tables 1 and 2). The effect of different post systems on the fracture resistance of the tooth was assessed by comparison of groups using ANOVA test and post hoc Tukey HSD test (Tables 3 and 4). In the above tests, $p \leq 0.05$ was taken to be statistically significant. All analyses were performed using Statistical Package for the Social Science software, version 17. 
Fracture Resistance of Endodontically Treated Teeth Restored Using Three Different Esthetic Post Systems

Table 1: Comparison of force required to cause fracture under $90^{\circ}$ vertical load

\begin{tabular}{lll}
\hline Group & Mean + standard deviation & p value \\
\hline Rebilda post GT system & $302.69 \pm 3.76$ & 0.000 \\
EverStick fiber post & $205.63 \pm 2.83$ & 0.000 \\
Prefabricated fiber post & $194.97 \pm 2.70$ & 0.000 \\
Control & $157.90 \pm 3.83$ & 0.000 \\
\hline
\end{tabular}

Since $p$ value for the ANOVA test is less than that of 0.05 indicates significance of difference

Table 2: Comparison of force required to cause fracture under $45^{\circ}$ oblique load

\begin{tabular}{lll}
\hline Group & Mean & Standard deviation \\
\hline Rebilda post GT system & 265.65 & 4.67 \\
EverStick fiber post & 183.73 & 5.34 \\
Prefabricated fiber post & 175.34 & 3.61 \\
Control & 116.63 & 5.26 \\
\hline
\end{tabular}

Since $p$ value for the ANOVA test is less than that of 0.05 indicates significance of difference

Table 3: Post hoc Tukey HSD test to evaluate significance of difference under $90^{\circ}$ vertical load

\begin{tabular}{llccl}
\hline Group I & Group II & Mean difference (I-J) & Std. error & p value \\
\hline Rebilda post GT system & EverStick fiber post & 97.05700 & 1.48511 & 0.000 \\
Rebilda post GT system & Prefabricated fiber post & 107.71500 & 1.48511 & 0.000 \\
Rebilda post GT system & Control & 144.78600 & 1.48511 & 0.000 \\
EverStick fiber post & Prefabricated fiber post & 10.65800 & 1.48511 & 0.000 \\
EverStick fiber post & Control & 47.72900 & 1.48511 & 0.000 \\
Prefabricated fiber post & Control & 37.07100 & 1.48511 & 0.000 \\
\hline
\end{tabular}

$p$ value less than that of 0.05 indicates significance of difference and positive value in mean difference indicates higher fracture loading average in group I than group II

Table 4: One-way ANOVA with post hoc Tukey HSD test to evaluate the significance of differences under a $45^{\circ}$ oblique load

\begin{tabular}{llccc}
\hline Group I & Group II & Mean difference $(I-J)$ & Std. error & $p$ value \\
\hline Rebilda post GT system & EverStick fiber post & 81.91600 & 2.13376 & 0.000 \\
Rebilda post GT system & Prefabricated fiber post & 90.31300 & 2.13376 & 0.000 \\
Rebilda post GT system & Control & 149.01300 & 2.13376 & 0.000 \\
EverStick fiber post & Prefabricated fiber post & 8.39700 & 2.13376 & 0.002 \\
EverStick fiber post & Control & 67.09700 & 2.13376 & 0.000 \\
Prefabricated fiber post & Control & 58.70000 & 2.13376 & 0.000 \\
\hline
\end{tabular}

A $p$ value less than that of 0.05 indicates significance of difference and positive value in mean difference indicates higher fracture loading average in group I than group II

\section{RESULTS}

The samples were evaluated by subjecting them to two different loads, $90^{\circ}$ and $45^{\circ}$. Under the $90^{\circ}$ vertical as well as $45^{\circ}$ oblique loads, group I-Rebilda post GT samples fractured at a force of $306.29 \mathrm{~N}$ and $265.65 \mathrm{~N}$ respectively, showing maximum fracture resistance, followed by the EverStick fiber post group, prefabricated post and least in the control group.

\section{Discussion}

It is well established that dentin exhibits a fracture toughening mechanism, thereby reducing the possibility of crack progression. ${ }^{4}$ For these reasons, a minimally invasive post space preparation protocol was performed. The results of the study revealed that the fracture resistance of the tooth differed significantly between different prefabricated glass fiber post systems. In general, improvements in the fracture resistance values $(N)$ were found in the following order: Rebilda post GT > EverStick > Prefabricated glass fiber post $>$ Control.

Rebilda post GT (VOCO GmbH, Germany) (group I) system showed the maximum resistance against vertical as well as oblique load. This could be attributed to a better chemical bond between the glass fiber and the resin matrix. Also, increase in number of fibers in the coronal aspect leads to better bonding to the core and thus better stress distribution. ${ }^{15}$ In addition, Maceri et al., ${ }^{16}$ investigated single, double, and triple prefabricated composite post under several loads and indicated multi-post solution induces a significant reduction of stress levels into the residual dentin and therefore the root fracture risk decreases as well as the expected polymerization shrinkage effects. This could be accounted for the higher resistance exhibited by Rebilda post GT.

EverStick post (group II) showed better fracture resistance than Prefabricated post. EverStick post system allows the additional number of unpolymerized posts to be added according to the canal 
morphology, which leads to better adaptation and better stress distribution. The results of the current study are in accordance with Sorensen et al., who concluded that a significant increase could be measured in the fracture resistance of restored teeth when the posts are adapted closely to the canal walls. ${ }^{17}$ The root canal completely filled with fibers is a more effective reinforcement than one post only when compared under the same polymerization procedure. ${ }^{18}$

Through the use of a multi-post technique utilizing small diameter posts, one is able to fill large and irregular root cavities more efficiently than with a single, centrally positioned post. ${ }^{17}$

Single prefabricated glass fiber post (group III) showed the least fracture resistance in comparison to other glass fiber post systems. As stated by Hatta et al., this could be attributed to the thick cement layer resulting in weakened reinforcement. ${ }^{18}$ Prefabricated post with poor adaptation to oval canals undergoes flexure to functional stress and produce micro-movement of the core. ${ }^{19}$

Fracture of the post(s) or the restored tooth itself is among the most common failures of ETT restoration. ${ }^{20}$ In the present study, no difference was found between the study groups regarding the fracture pattern. The results of the present study revealed no catastrophic root fractures and were in agreement with Sirimai and Sidoli demonstrating no root fractures in teeth restored, with fiber posts, i.e., restorable fractures (above CEJ). ${ }^{21}$

The tested specimens received a vertical and oblique load (90degree and 45-degree to the long axis of the tooth, respectively). From the present study, it could be concluded that posts significantly contributed to the reinforcement and strengthening of decoronated pulpless teeth by supporting the remaining tooth structure against vertical compressive force. As described by Wandscher et al., oblique load appears to be the worst-case scenario in terms of the fracture resistance of ETT. ${ }^{22}$ Applying this angle of force to teeth without a dental ferrule resulted in significant stress on the cervical aspect of the restored tooth ${ }^{23}$ and heavy shear forces on the post/luting agent/radicular dentin interfaces.

The data demonstrate low fracture loads across the samples that can be attributed to the compromised tooth structure (no ferrule), unfavorable loading forces, and a lack of crown restoration. This highlights the importance of these three factors in the clinical success of restored, endodontically treated teeth and the importance of further investigations to achieve meaningful information about the best way to restore these teeth clinically.

\section{Conclusion}

- The decoronated endodontically treated teeth without postcore system showed the least fracture resistance demonstrating the need to reinforce the tooth.

- Use of Rebilda post GT and EverStick post reinforce the tooth structure better as compared to Prefabricated post.

- No root fractures were seen any of the teeth restored with glass fiber posts, making them more amenable to retreatment.

\section{References}

1. Saritha MK, Paul U, et al. Comparative evaluation of fracture resistance of different post systems. J Int Soc Prev Community Dent 2017 Nov;7(6):356-359. DOI: 10.4103/jispcd.JISPCD_413_17.

2. Nam SH, Chang HS, et al. Effect of the number of residual walls on fracture resistances, failure patterns, and photoelasticity of simulated premolars restored with or without fiber-reinforced composite posts. J Endod 2010;36:297-301. DOI: 10.1016/j.joen.2009.10.010.
3. Dietschi D, Duc O, et al. Biomechanical considerations for the restoration of endodontically treated teeth: a systematic review of the literature-Part 1. Composition and micro and macrostructure alterations. Quintessence Int 2007;38:733-743.

4. Al-Omiri MK, Mahmoud AA, et al. Fracture resistance of teeth restored with post-retained restorations: an overview. J Endod 2010;36: 1439-1449. DOI: 10.1016/j.joen.2010.06.005.

5. Clark D, Khademi J. Modern molar endodontic access and directed dentin conservation. Dent Clin North Am 2010;54:249-273. DOI: 10.1016/j.cden.2010.01.001.

6. Schwartz RS, Robbins JW. Post placement and restoration of endodontically treated teeth: a literature review. J Endod 2004;30:289-301. DOI: 10.1097/00004770-200405000-00001.

7. Cecchin D, Farina AP, et al. Fracture resistance of roots prosthetically restored with intra-radicular posts of different lengths. J Oral Rehabil 2010;37:116-122. DOI: 10.1111/j.1365-2842.2009.02028.x.

8. Mitsui FH, Marchi GM, et al. In vitro study of fracture resistance of bovine roots using different intra radicular post systems. Quintessence Int 2004;35:612-616.

9. Zicari F, Van Meerbeek B, et al. Effect of fiber post length and adhesive strategy on fracture resistance of endodontically treated teeth after fatigue loading. JDent 2012;40:312-321. DOI: 10.1016/j.jdent.2012.01.006.

10. Stricker EJ, Göhring TN. Influence of different posts and cores on marginal adaptation, fractures resistance, and fracture mode of composite resin crowns on human mandibular premolars: An in vitro study. J Dent 2006;34:326-335. DOI: 10.1016/j.jdent.2005.07.007.

11. Newmann MP, Yaman P, et al. Fracture resistance of endodontically treated teeth restored with composite posts. J Prosthet Dent 2003;89:360-367. DOI: 10.1067/mpr.2003.75.

12. Silva NR, Castro CG, et al. Influence of different post design and composition on stress distribution in maxillary central incisor: Finite element analysis. Indian J Dent Res 2009;20(2):153-158.

13. Cooney JP, Caputo AA, et al. Retention and stress distribution of tapered-end endodontic posts. J Prosthet Dent 1986;55:540-546. DOI: 10.1016/0022-3913(86)90028-4.

14. Lassila LV, Tanner J, et al. Flexural properties of fiber reinforced root canal posts. Dent Mater 2004;20:29-36. DOI: 10.1016/S01095641(03)00065-4.

15. Galhano GA, Valandro LF, et al. Evaluation of the flexural strength of carbon fiber-, quartz fiber-, and glass fiber-based posts. J Endod 2005;31:209-211. DOI: 10.1097/01.don.0000137652.49748.0c.

16. Maceri $F$, Martignoni M, et al. Mechanical behaviour of endodontic restorations with multiple prefabricated posts: a finite-element approach. J Biomech 2007;40:2386-2398. DOI: 10.1016/j.jbiomech. 2006.11.018.

17. Sorensen JA, Engelman MJ. Effect of post adaptation on fracture resistance of endodontically treated teeth. J Prosthet Dent 1990;64:419-424. DOI: 10.1016/0022-3913(90)90037-D.

18. Hatta $M$, Shinya $A$, et al. High volume individual fiber post versus low volume fiber post: the fracture load of the restored tooth. J Dent 2011;39:65-71. DOI: 10.1016/j.jdent.2010.10.004.

19. Pantaleón DS, Morrow BR, et al. Influence of remaining coronal tooth structure on fracture resistance and failure mode of restored endodontically treated maxillary incisors. J Prosthet Dent 2018 Mar 1;119(3):390-396. DOI: 10.1016/j.prosdent.2017.05.007.

20. Fuss Z, Lustig J, et al. Prevalence of vertical root fractures in extracted endodontically treated teeth. Int Endod J 1999;32:283-286. DOI: 10.1046/j.1365-2591.1999.00208.x.

21. Sirimai $\mathrm{S}$, Riis $\mathrm{DN}$, et al. An in vitro study of the fracture resistance and the incidence of vertical root fracture of pulpless teeth restored with six post-and-core systems. J Prosthet Dent 1999;81:262-269. DOI: 10.1016/S0022-3913(99)70267-2.

22. Wandscher VF, Bergoli CD, et al. Preliminary results of the survival and fracture load of roots restored with intracanal posts: weakened vs nonweakened roots. Oper Dent 2014;39:541-555. DOI: 10.2341/12-465.

23. Meira JB, Esposito CO, et al. Elastic modulus of posts and the risk of root fracture. Dent Traumatol 2009;25:394-398. DOI: 10.1111/j.16009657.2009.00772.x. 